\title{
Quark-meson coupling model with the cloudy bag
}

\author{
S. Nagai, T. Miyatsu, and K. Saitd* \\ Department of Physics, Faculty of Science and Technology, \\ Tokyo University of Science, Noda 278-8510, Japan \\ K. Tsushima \\ Excited Baryon Analysis Center (EBAC), Theory Group, \\ Thomas Jefferson National Accelerator Facility \\ 12000 Jefferson Avenue, Newport News, VA 23606, USA
}

(Dated: November 6, 2018)

\begin{abstract}
Using the volume coupling version of the cloudy bag model, the quark-meson coupling model is extended to study the role of pion field and the properties of nuclear matter. The extended model includes the effect of gluon exchange as well as the pion-cloud effect, and provides a good description of the nuclear matter properties. The relationship between the extended model and the EFT approach to nuclear matter is also discussed.
\end{abstract}

PACS numbers: 12.39.Ba, 21.65.-f, 12.39.Ki

Keywords: quark-meson coupling model, nuclear matter, cloudy bag, chiral symmetries

*Electronic address: ksaito@ph.noda.tus.ac.jp 
The quark-meson coupling (QMC) model [1] can be considered as an extension of Quantum Hadrodynamics (QHD) to include the effect of the internal structure of a nucleon in matter. The model describes a nuclear system by non-overlapping MIT bags, in which the confined quarks interact through the self-consistent exchange of isoscalar, scalar $(\sigma)$ and vector $(\omega)$ mesons. In the past few decades, it has been extensively developed and applied to various nuclear phenomena with tremendous success [1].

On the other hand, a major breakthrough occurred in the problem of nucleon-nucleon (NN) force by introducing the concept of an effective field theory (EFT) [2]. The QCD Lagrangian for massless up and down quarks is chirally symmetric, and the axial symmetry is spontaneously broken. This implies the existence of the massless Nambu-Goldstone bosons, namely the pions. The non-zero pion mass is then a consequence of the fact that the light quark has a small mass. Thus, one arrives at a low-energy scenario that pions and nucleons (and possibly deltas) interact via a force governed by spontaneously broken, approximate chiral symmetry.

In EFT, the degrees of freedom of quarks and gluons (including heavy mesons and nucleon resonances) should be integrated out (or cutoff), because a probe with wavelength $\lambda$ is insensitive to details of structure at distances much smaller than it [3]. Instead, it is necessary to add local (contact) interactions with low-energy constants (LECs) to the Lagrangian to mimic the effect of the true short distance physics. The LECs are determined empirically from fits to $\pi \mathrm{N}$ and/or NN scattering data, and vary with the momentum cutoff $\left(\sim \lambda^{-1}\right)$ accounting for quantum fluctuations excluded by the cutoff [3]. Up to $\mathrm{N}^{3} \mathrm{LO}$, EFT can provide the peripheral NN scattering data (below about $250 \mathrm{MeV}$ lab. energy) very accurately [4].

Recently, EFT has been intensively applied to the problem of nuclear matter. In addition to the usual (small momentum) expansion in the free $\mathrm{NN}$ or $\pi \mathrm{N}$ scattering, physical observables in matter are expanded in terms of the Fermi momentum $k_{F}$, which is also a relevant, small scale. Such density dependence arises from the Pauli blocking effect in matter, i.e., the medium insertion including the step-function, $\theta\left(k_{F}-|\vec{p}|\right)$, in the nucleon propagator. Then, the strengths of the LECs are fine-tuned so as to reproduce the nuclear matter properties [5].

If the internal structure of the nucleon were completely frozen in a nuclear medium or the same as that in free space, it might be sufficient to consider the density dependence solely stemming from the Pauli blocking effect. However, if the in-medium nucleon were 
metamorphosed depending on the nuclear density $\rho_{B}$, the situation may be different. In fact, the evidence for the medium modification of nucleon structure was observed in polarization transfer measurement in the quasi-elastic $\left(e, e^{\prime} p\right)$ reaction at the Thomas Jefferson national accelerator facility, and the result supports the prediction of the QMC model [6]. It also seems vital to consider the internal structure change of the nucleon to understand the nuclear EMC effect [7].

The QMC model can describe the medium modification of the nucleon structure through the quark model, and predict the density (or mean scalar-field) dependence of physical quantities [1]. Expanding such modification in terms of $k_{F}$ and comparing with the values of LECs given in the EFT approach, ${ }^{1}$ it may be possible to study whether the internal structure change of a nucleon indeed shows up in matter, since the LECs involve all information on the short distance physics.

To carry out such a complicated investigation, as a first step, we need to develop a new version of the QMC model for nuclear matter, where the structure of nucleon (and delta) is treated based on chiral symmetry. We attempt this in the present study using the volume coupling version of the cloudy bag model (CBM), which incorporates major results of the current algebra for low energy $\pi \mathrm{N}$ scattering [9].

The Lagrangian density for the volume coupling version of the CBM in flavor $\mathrm{SU}(2)$ is given by [9]

$$
\mathcal{L}_{C B M}=\left[\bar{\psi}\left\{i \gamma_{\mu} \mathcal{D}^{\mu}+\frac{1}{2 f_{\pi}} \gamma_{\mu} \gamma_{5} \vec{\tau} \cdot\left(D^{\mu} \vec{\phi}\right)\right\} \psi-B\right] \theta_{V}-\frac{1}{2} \bar{\psi} \psi \delta_{S}+\frac{1}{2}\left(D_{\mu} \vec{\phi}\right)^{2}+\mathcal{L}_{\chi B},
$$

with $\psi$ the quark field, $\vec{\phi}$ the pion field, $\phi=(\vec{\phi} \cdot \vec{\phi})^{1 / 2}, \hat{\phi}=\vec{\phi} /|\vec{\phi}|, f_{\pi}(=93 \mathrm{MeV})$ the pion decay constant, $B$ the bag constant, $\theta_{V}$ the step function for the bag, $\delta_{S}$ the surface $\delta$-function, $D_{\mu} \vec{\phi}=\left(\partial_{\mu} \phi\right) \hat{\phi}+f_{\pi} \sin \left(\phi / f_{\pi}\right) \partial_{\mu} \hat{\phi}$ and $\mathcal{D}^{\mu} \psi=\partial^{\mu} \psi-\frac{i}{2}\left[\cos \left(\phi / f_{\pi}\right)-1\right] \vec{\tau} \cdot\left(\hat{\phi} \times \partial^{\mu} \hat{\phi}\right) \psi$. The last term includes the quark mass, $m$, which explicitly breaks chiral symmetry, and the pion mass, $m_{\pi}(=138 \mathrm{MeV}): \mathcal{L}_{\chi B}=-m \bar{\psi} e^{-i \vec{\tau} \cdot \vec{\phi} \gamma_{5} / f_{\pi}} \psi \theta_{V}-\frac{1}{2} m_{\pi}^{2} \vec{\phi}^{2}$.

As in the CBM, we linearize the pion field and keep $\mathcal{O}\left(1 / f_{\pi}\right)$ (the convergence properties

1 In fact, from the poin of view of the quark and gluon degrees of freedom, the QMC model can explain the values of the coefficients appearing in the familiar (contact) Skyrme force in conventional nuclear physics [8] . 
of the CBM were given in Ref. [9]). The Lagrangian density then reads

$$
\begin{aligned}
\mathcal{L}_{C B M} & =\left[\bar{\psi}\left\{i \gamma_{\mu} \partial^{\mu}-m+i \frac{m}{f_{\pi}} \gamma_{5} \vec{\tau} \cdot \vec{\phi}+\frac{1}{2 f_{\pi}} \gamma_{\mu} \gamma_{5} \vec{\tau} \cdot\left(\partial^{\mu} \vec{\phi}\right)\right\} \psi-B\right] \theta_{V}-\frac{1}{2} \bar{\psi} \psi \delta_{S} \\
& +\frac{1}{2}\left(\partial_{\mu} \vec{\phi}\right)^{2}-\frac{1}{2} m_{\pi}^{2} \vec{\phi}^{2} .
\end{aligned}
$$

Here the pion field interacts with the quark through both the pseudovector (pv) and pseudoscalar (ps) couplings. The strength of the ps coupling is $\mathcal{O}\left(m / f_{\pi}\right)$, which explicitly shows the breaking scale of chiral symmetry.

We introduce the gluon field as well. The resulting Lagrangian density is thus given by

$$
\mathcal{L}_{C B M}=\mathcal{L}_{B A G}+\mathcal{L}_{\pi}+\mathcal{L}_{g}+\mathcal{L}_{\text {int }}
$$

where

$$
\begin{gathered}
\mathcal{L}_{B A G}=\left[\bar{\psi}\left(i \gamma_{\mu} \partial^{\mu}-m\right) \psi-B\right] \theta_{V}-\frac{1}{2} \bar{\psi} \psi \delta_{S}, \\
\mathcal{L}_{\text {int }}=\bar{\psi}\left[i \frac{m}{f_{\pi}} \gamma_{5} \vec{\tau} \cdot \vec{\phi}+\frac{1}{2 f_{\pi}} \gamma_{\mu} \gamma_{5} \vec{\tau} \cdot\left(\partial^{\mu} \vec{\phi}\right)+\frac{g}{2} \gamma_{\mu} \vec{\lambda} \cdot \vec{A}^{\mu}\right] \psi \theta_{V},
\end{gathered}
$$

with $\vec{\lambda}$ the SU(3) generators and $g$ the quark-gluon coupling constant. The free pion field and the kinetic energy of the gluon field, $\overrightarrow{A^{\mu}}$, are, respectively, described by $\mathcal{L}_{\pi}$ and $\mathcal{L}_{g}$.

We firstly calculate the second-order energy correction to the nucleon or delta mass. The energy shift of a multi-quark, ground state, $|0\rangle$, due to the interaction is given by the Hubbard's prescription

$$
E-E_{0}=\left\langle 0\left|\sum_{m=1}^{\infty}(-i)^{m} \frac{1}{m !} \int i \delta\left(t_{1}\right) d^{4} x_{1} \cdots \int d^{4} x_{m} T\left[\mathcal{H}_{\text {int }}\left(x_{1}\right) \cdots \mathcal{H}_{\text {int }}\left(x_{m}\right)\right]\right| 0\right\rangle_{\text {con. }}
$$

where $\mathcal{H}_{\text {int }}$ is the interaction Hamiltonian density. The energy shift is then given as $E^{(2)}=$ $E_{d r}+E_{n d}$, where the first term is the direct contribution and the second one is the non-direct contribution. (See Eqs.(11) and (12) later.)

The noninteracting, quark green function is given by $i G^{0}\left(r, r^{\prime}\right)=\left\langle 0\left|T\left[\psi(r) \bar{\psi}\left(r^{\prime}\right)\right]\right| 0\right\rangle$, and it can be separated into two pieces: $G^{0}\left(r, r^{\prime}\right)=\int \frac{d \omega}{2 \pi} e^{-i \omega\left(t-t^{\prime}\right)}\left[G_{F}^{0}\left(\vec{r}, \vec{r}^{\prime}, \omega\right)+G_{D}^{0}\left(\vec{r}, \vec{r}^{\prime}, \omega\right)\right]$. The first term is the usual Feynman propagator in a spherical cavity (bag) and the second one describes the occupied, multi-quark ground state [10]

$$
G_{D}^{0}\left(\vec{r}, \vec{r}^{\prime}, \omega\right)=\sum_{n \leq n_{F}} U_{n}(\vec{r}) \bar{U}_{n}\left(\vec{r}^{\prime}\right) 2 \pi i \delta\left(\omega-E_{n}\right)
$$


where $U_{n}$ is the positive energy state with a complete set of quark quantum numbers $n(=$ $\left.\left\{\nu \kappa \mu \mu_{i} \mu_{c}\right\}\right)$ including isospin $\mu_{i}$ and color $\mu_{c}$ ( $n_{F}$ specifies the quantum numbers at the Fermi surface in a hadron).

Here we restrict the expansion of the quark propagator to the ground state, i.e., $\nu=0$ and $\kappa=-1$. Such a truncation may be considered as a regularization of the quark propagator, where in flavor $\mathrm{SU}(2)$ the intermediate baryon states in loop diagrams are restricted to the nucleon and delta [11]. This is consistent with the idea of the CBM. Thus, we let $n$ label the spin, isospin and color $\left\{\mu \mu_{i} \mu_{c}\right\}$.

The pion propagator is defined by $i \Delta_{a b}\left(r, r^{\prime}\right)=\left\langle 0\left|T\left[\phi_{a}(r) \phi_{b}\left(r^{\prime}\right)\right]\right| 0\right\rangle=i \delta_{a b} \Delta\left(r, r^{\prime}\right)$, where $(a, b)$ specifies the isospin. It is then given by the multipole expansion

$$
\Delta\left(r, r^{\prime}\right)=\sum_{\ell, m} \int \frac{d \omega}{2 \pi} e^{-i \omega\left(t-t^{\prime}\right)} \Delta_{\ell}\left(r, r^{\prime}, \omega\right) Y_{\ell m}(\hat{r}) Y_{\ell m}^{*}\left(\hat{r}^{\prime}\right) .
$$

The gluon propagator can be calculated in the Coulomb gauge ${ }^{2}$

$$
\begin{aligned}
& i D_{00}^{c d}\left(r, r^{\prime}\right)=i \delta_{c d} \int \frac{d^{4} k}{(2 \pi)^{4}} \frac{e^{-i k \cdot\left(x-x^{\prime}\right)}}{\vec{k}^{2}}=i \delta_{c d} D_{00}\left(r, r^{\prime}\right), \\
& i D_{i j}^{c d}\left(r, r^{\prime}\right)=i \delta_{c d} \int \frac{d^{4} k}{(2 \pi)^{4}} \frac{e^{-i k \cdot\left(x-x^{\prime}\right)}}{\vec{k}^{2}+i \epsilon}\left(\delta_{i j}-\frac{k_{i} k_{j}}{\vec{k}^{2}}\right)=i \delta_{c d} D_{i j}\left(r, r^{\prime}\right),
\end{aligned}
$$

where $D_{00}^{c d}\left(D_{i j}^{c d}\right)$ represents the Coulomb (transverse) propagator, $(i, j)=1,2,3$ and $(c, d)$ specifies the color.

Using these propagators, the direct (or Hartree) contribution between two quarks is calculated by

$$
E_{d r}=-\frac{1}{2} \iint \delta\left(t_{1}\right) d^{4} r_{1} d^{4} r_{2} \Theta\left(r_{1}, r_{2}\right) \operatorname{tr}\left[\Gamma_{1} \vec{t}_{1} G_{D}^{0}\left(r_{1}, r_{1}\right)\right] \cdot \operatorname{tr}\left[\Gamma_{2} \vec{t}_{2} G_{D}^{0}\left(r_{2}, r_{2}\right)\right],
$$

where $\Gamma_{1,2}$ represents the vertex (ps, pv or gluon) including the coupling constant (the Lorentz index is suppressed here), $\Theta$ stands for the pion or gluon propagator and $\vec{t}=\vec{\tau} / 2$ or $\vec{\lambda} / 2$. The non-direct contribution is given by

$$
E_{n d}=\frac{1}{2} \iint \delta\left(t_{1}\right) d^{4} r_{1} d^{4} r_{2} \Theta\left(r_{1}, r_{2}\right) \operatorname{tr}\left[\Gamma_{1} \vec{t}_{1} G^{0}\left(r_{1}, r_{2}\right) \cdot \Gamma_{2} \vec{t}_{2} G^{0}\left(r_{2}, r_{1}\right)\right] .
$$

This consists of the exchange contribution, $E_{e x}$, which is evaluated with $G^{0}=G_{D}^{0}$ in Eq.(12), and the self-energy contribution, $E_{q s f}$, where the pion is emitted and absorbed by the same

\footnotetext{
$\overline{2}$ It can be shown that the result does not depend on the choice of the gauge [11].
} 
quark. $E_{q s f}$ is calculated by replacing one of $G_{D}^{0}$ 's with $G_{F}^{0}$ in $E_{e x}$. The case with simultaneously $G^{0}=G_{F}^{0}$ is removed, because it is the vacuum energy. The Hartree-Fock (HF) contribution between two quarks is defined by $E_{q H F}=E_{d r}+E_{e x}$. Note that these energy shifts correspond to the case where a closure approximation to the intermediate states is taken in the CBM, and that such an approximation is reasonable.

For example, the HF result for the pv coupling, $E_{q H F}^{p v}$, is given by

$$
\begin{aligned}
E_{q H F}^{p v} & =-\frac{[\vec{\sigma} \vec{t}]_{H F} N^{4}}{12 \pi^{2} x^{3}\left(f_{\pi} R\right)^{2} R} \int_{0}^{x} d \rho_{1} \rho_{1}^{2} \int_{0}^{x} d \rho_{2} \rho_{2}^{2} \int_{0}^{\infty} \frac{d t t^{4}}{t^{2}+y^{2}}\left[A\left(\rho_{1}\right) A\left(\rho_{2}\right) j_{0}\left(t \rho_{1}\right) j_{0}\left(t \rho_{2}\right)\right. \\
& +\frac{1}{3}\left\{A\left(\rho_{1}\right) B\left(\rho_{2}\right) j_{0}\left(t \rho_{1}\right)\left(j_{0}\left(t \rho_{2}\right)-2 j_{2}\left(t \rho_{2}\right)\right)+A\left(\rho_{2}\right) B\left(\rho_{1}\right) j_{0}\left(t \rho_{2}\right)\left(j_{0}\left(t \rho_{1}\right)-2 j_{2}\left(t \rho_{1}\right)\right)\right\} \\
& \left.+\frac{1}{9} B\left(\rho_{1}\right) B\left(\rho_{2}\right)\left(j_{0}\left(t \rho_{1}\right)-2 j_{2}\left(t \rho_{1}\right)\right)\left(j_{0}\left(t \rho_{2}\right)-2 j_{2}\left(t \rho_{2}\right)\right)\right],
\end{aligned}
$$

with $N$ the normalization constant for the quark wave function, $R$ the bag radius, $x$ the lowest quark eigenvalue, $y=m_{\pi} R / x, A(\rho)=j_{0}^{2}(\rho)-\beta^{2} j_{1}^{2}(\rho), B(\rho)=2 \beta^{2} j_{1}^{2}(\rho), \beta=$ $x /(\alpha+\delta), \alpha^{2}=x^{2}+\delta^{2}$ and $\delta=m R$. Here the spin-isospin matrix element is given by [10]

$$
[\vec{\sigma} \vec{t}]_{H F}=\sum_{i \neq i^{\prime} \in N, \Delta}\left\langle i|\vec{\sigma} \vec{t}| i^{\prime}\right\rangle \cdot\left\langle i^{\prime}|\vec{\sigma} \vec{t}| i\right\rangle=9-S(S+1)-I(I+1),
$$

where the index $\left(i, i^{\prime}\right)$ runs over the spin and isospin, and $S(I)$ is the total spin (isospin) of $\mathrm{N}$ or $\Delta$. Because the intermediate baryon states are restricted to the lowest mode, the self-energy contribution, $E_{q s f}^{p v}$, has the same form as the HF result except for the spin-isospin matrix element: $[\vec{\sigma} \vec{t}]_{s f}=27 / 4$ for both $\mathrm{N}$ and $\Delta$.

The pion-induced baryon self-energies should reproduce the correct, leading non-analytic (LNA) behavior of chiral perturbation theory $(\chi \mathrm{PT})[12]$. The LNA contribution is associated with the infrared behavior of the baryon self-energy, and hence, for example, Eq.(13) gives $-3 g_{A}^{2} m_{\pi}^{3} /\left(32 \pi f_{\pi}^{2}\right) \times(30 / 25,6 / 25)$ for the $(\mathrm{N}, \Delta)$ in the infrared limit. In contrast, $E_{q s f}^{p v}$ gives $-3 g_{A}^{2} m_{\pi}^{3} /\left(32 \pi f_{\pi}^{2}\right) \times 27 / 25$ for both $\mathrm{N}$ and $\Delta$. Thus, the total amount is $-3 g_{A}^{2} m_{\pi}^{3} /\left(32 \pi f_{\pi}^{2}\right) \times(57 / 25,33 / 25)$ for the $(\mathrm{N}, \Delta)$, which is precisely the leading-order correction given by large $N_{c} \chi \mathrm{PT}$. However, because of the closure approximation taken for the intermediate states, the term of $m_{\pi}^{4} \ln \left(m_{\pi}\right)$ does not appear in the present calculation.

The contribution from the ps (gluon) interaction, $E_{q H F, q s f}^{p s}\left(E_{q H F, q s f}^{g}\right)$, is also calculated in the similar manner. ${ }^{3}$ Note that there exists a nonvanishing, interference (sv) contribution

3 The energy shift due to the Coulomb propagator vanishes because of the color charge neutrality [13]. 
between the ps and pv couplings, $E_{q H F, q s f}^{s v}$. The pv, ps and gluon corrections lower the baryon mass, while the interference contribution increases it but its magnitude is $\mathcal{O}\left(m / f_{\pi}^{2} R\right)$ and thus small.

Each correction is a function of the bag radius $R$, and, for example, $E_{q H F}^{p v}$ diverges like $\sim-1 / R^{3}$ as $R \rightarrow 0$. Thus, the bag collapses as $R \rightarrow 0$. Because the pion has a finite size, the effect of the $q \bar{q}$ substructure is essentially important when the bag radius is very small. In Ref. [14], a phenomenological, non-local interaction was studied to settle this collapse at $R \sim 0$. The effect of the $q \bar{q}$ substructure of pion can eventually be described by a form factor at the vertex of the quark-pion interaction. When the charge radius of the pion is about $0.56 \mathrm{fm}[15]$, the form factor is estimated as

$$
F_{q \pi}(R)=\frac{1}{\left(1+1.3 \times(b / R)^{2}\right)^{3 / 2}},
$$

with $b=0.46 \mathrm{fm}$ (see Fig.2 in Ref.[14]). Using this form factor, one can get the finite, pion-loop contributions.

The coupling between a quark and gluon is scale-dependent and the lowest-order coupling at momentum transfer $Q^{2}$ is $\alpha_{s}\left(Q^{2}\right)=g^{2} / 4 \pi=12 \pi /\left[\left(33-2 N_{f}\right) \ln \left(Q^{2} / \Lambda_{Q C D}^{2}\right)\right]$ with $N_{f}$ quark flavors and $\Lambda_{Q C D} \simeq 200 \mathrm{MeV}$. In practice, this behavior can be parametrized in a convenient form [16]

$$
\alpha_{s}\left(Q^{2}\right)=\sum_{k} \alpha_{k} e^{-Q^{2} / 4 \gamma_{k}^{2}}=a_{1} e^{-4 Q^{2}}+0.25 e^{-Q^{2}}+0.15 e^{-Q^{2} / 10}+0.2 e^{-Q^{2} / 1000},
$$

where $Q^{2}$ in $\mathrm{GeV}^{2}$ and the parameters, $\alpha_{k}$ and $\gamma_{k}$, except $a_{1}$ are constrained to follow the behavior of $\alpha_{s}\left(Q^{2}\right)$. We treat $a_{1}$ as a parameter. The form (16) is convenient, because it is easily transformed into the form in coordinate space

$$
\alpha_{s}(R)=\sum_{k} \frac{2 \alpha_{k}}{\sqrt{\pi}} \int_{0}^{\gamma_{k} R} e^{-x^{2}} d x .
$$

Here we assume that this gives the coupling constant at the scale of the bag radius, $R$, and that the energy shift due to the gluon exchange is given by replacing $g^{2}$ with $4 \pi \alpha_{s}(R)$ in $E_{q H F, q s f}^{g}(R)$. Note that $\alpha_{s}(R) \rightarrow 0$ as $R \rightarrow 0$.

Now we are in a position to present the numerical result for the $\mathrm{N}$ or $\Delta$ mass in free space. The mass is given by a sum of the usual bag energy [13] and the corrections due to the pion and gluon exchanges. We fix the current quark mass $m=5 \mathrm{MeV}$, because 


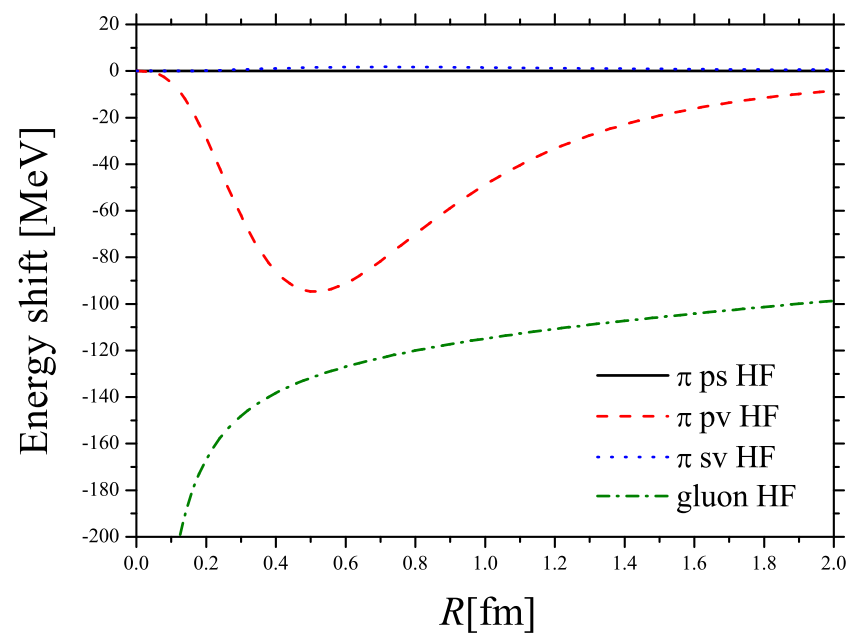

FIG. 1: Energy shift due to the HF contribution $\left(R_{N}=0.8 \mathrm{fm}\right)$. The dot-dashed curve presents the gluon contribution, which approaches a constant as $R \rightarrow 0$. The solid (dashed) [dotted] curve is for the ps (pv) $[\mathrm{sv}]$ contribution from the pion exchange.

the dependence of the baryon mass on $m$ is very weak. There are four parameters: $B, z_{N}$, $z_{\Delta}$ and $a_{1}\left(\right.$ in $\left.\alpha_{s}\right)$. Since we can expect that the usual $z$ parameter for the $\mathrm{N}$ is not much different from that for the $\Delta$, we choose $z_{0}=z_{N}=z_{\Delta}$. Then, the bag constant, $B$, and $z_{0}$ are determined so as to fit the free nucleon mass, $M_{N}(=939 \mathrm{MeV})$, with its radius $R_{N}=0.6$ or $0.8 \mathrm{fm}$. The remaining parameter, $a_{1}$, is fixed so as to yield the correct mass difference between $M_{N}$ and $M_{\Delta}(=1232 \mathrm{MeV})$ together with the pion-cloud contribution. We then find $B^{1 / 4}=231.8(183.7) \mathrm{MeV}, z_{0}=2.46(1.17)$ and $a_{1}=5.01(6.08)$ for $R_{N}=0.6(0.8) \mathrm{fm}$.

TABLE I: Energy shift (in MeV) due to the pion or gluon exchange.

\begin{tabular}{cccccc}
\hline \hline & $R(\mathrm{fm})$ & $E_{q H F}^{p v+p s+s v}$ & $E_{q s f}^{p v+p s+s v}$ & $E_{q H F}^{g}$ & $E_{q s f}^{g}$ \\
\hline $\mathrm{N}$ & 0.6 & -89.5 & -80.5 & -111.1 & -333.3 \\
$\Delta$ & 0.611 & -17.7 & -79.7 & 110.7 & -332.0 \\
\hline $\mathrm{N}$ & 0.8 & -68.3 & -61.5 & -120.0 & -360.1 \\
$\Delta$ & 0.823 & -13.7 & -59.1 & 119.4 & -358.1 \\
\hline \hline
\end{tabular}

In Table I, we present the energy corrections. The $\mathrm{N}-\Delta$ mass difference mainly comes from the gluon-exchange $\mathrm{HF}$ contribution. Note that the $\mathrm{N}-\Delta$ mass difference due to the pion cloud is about $60 \mathrm{MeV}$ (for $R_{N}=0.8 \mathrm{fm}$ ), and that is near the upper limit allowed 
from lattice QCD constraints [17]. In Fig. 1, for example, we show the HF energy due to the pion or gluon exchange as a function of the bag radius. As expected, the ps contribution is quite small. The interference is also small and its sign is positive. Because of Eqs.(15) and (17), the energy shift is finite everywhere and thus the total energy for the $\mathrm{N}$ or $\Delta$ mass has (global) one minimum at a certain $R$.

To describe a nuclear matter, we need the intermediate attractive and short-range repulsive nuclear forces. As the QMC model is based on the one-boson-exchange (OBE) picture [1], it is achieved by introducing the $\sigma$ and $\omega$ mesons. ${ }^{4}$ However, the present $\sigma$ meson is chirally singlet and not the chiral partner of the $\pi$ meson. This $\sigma$ represents, in some way, the exchange of two pions in the iso-scalar channel [18].

Now let us start from the following Lagrangian density for the "chiral quark-meson coupling (CQMC) model": $\mathcal{L}_{C Q M C}=\mathcal{L}_{C B M}+\mathcal{L}_{\sigma \omega}$, where

$$
\mathcal{L}_{\sigma \omega}=\bar{\psi}\left[g_{\sigma}^{q} \sigma-g_{\omega}^{q} \gamma_{0} \omega\right] \psi \theta_{V}-\frac{1}{2} m_{\sigma}^{2} \sigma^{2}+\frac{1}{2} m_{\omega}^{2} \omega^{2}
$$

with $g_{\sigma}^{q}\left(g_{\omega}^{q}\right)$ the $\sigma(\omega)$-quark coupling constant, $m_{\sigma}\left(m_{\omega}\right)$ the meson mass and $\sigma(\omega)$ the meanfield value of the $\sigma(\omega)$ meson.

In an iso-symmetric nuclear matter, the total energy per nucleon is given by

$$
E_{t o t}=\frac{4}{(2 \pi)^{3} \rho_{B}} \int^{k_{F}} d \mathbf{k} \sqrt{\mathbf{k}^{2}+M_{N}^{* 2}}+3 g_{\omega}^{q} \omega+\frac{1}{2}\left(m_{\sigma}^{2} \sigma^{2}-m_{\omega}^{2} \omega^{2}\right),
$$

with $\rho_{B}=2 k_{F}^{3} / 3 \pi^{2}$ and $M_{N}^{*}$ the effective nucleon mass. The attractive force due to the $\sigma$ changes the quark mass in matter as $m^{*}=m-g_{\sigma}^{q} \sigma$ ( $m^{*}$ the effective quark mass), which modifies the quark wave function. This modification generates the effective nucleon mass, $M_{N}^{*}$, in matter. Because the change of the quark wave function varies the source of the $\sigma$ field, we have to solve the coupled, nonlinear equations for the nuclear matter self-consistently (for details, see Refs.[1]).

The numerical result for the nuclear matter (with $m_{\sigma}=550 \mathrm{MeV}, m_{\omega}=783 \mathrm{MeV}$ and $R_{N}=0.6$ or $0.8 \mathrm{fm}$ ) is presented in Table II. The $\sigma-\mathrm{N}$ and $\omega-\mathrm{N}$ coupling constants, $g_{\sigma}\left(=3 g_{\sigma}^{q} S(\sigma=0)\right)$ and $g_{\omega}\left(=3 g_{\omega}^{q}\right)$, are determined to fit the nuclear saturation condition $(-15.7 \mathrm{MeV})$ at normal nuclear density $\rho_{0}\left(=0.15 \mathrm{fm}^{-3}\right)$. Here $S(\sigma)$ is the scalar density calculated by the quark wave function [1].

4 It should be noticed that the OBE model is still the most economical and quantitative phenomenology for describing the nuclear force [2]. 
TABLE II: Coupling constants and calculated properties for symmetric nuclear matter at $\rho_{0}$. The last three columns show the relative changes (from their values at zero density) of the bag radius, the lowest eigenvalue and the root-mean-square (rms) radius of the nucleon calculated with the quark wave function. The nucleon mass and the nuclear incompressibility, $K$, are in MeV.

\begin{tabular}{cccccccc}
\hline \hline$R_{N}(\mathrm{fm})$ & $g_{\sigma}^{2} / 4 \pi$ & $g_{\omega}^{2} / 4 \pi$ & $M_{N}^{*}$ & $K$ & $\delta R_{N}^{*} / R_{N}$ & $\delta x^{*} / x$ & $\delta r^{*} / r$ \\
\hline 0.6 & 6.11 & 10.20 & 632 & 362 & 0.01 & -0.18 & 0.05 \\
0.8 & 4.93 & 9.59 & 647 & 365 & 0.02 & -0.22 & 0.07 \\
\hline \hline
\end{tabular}

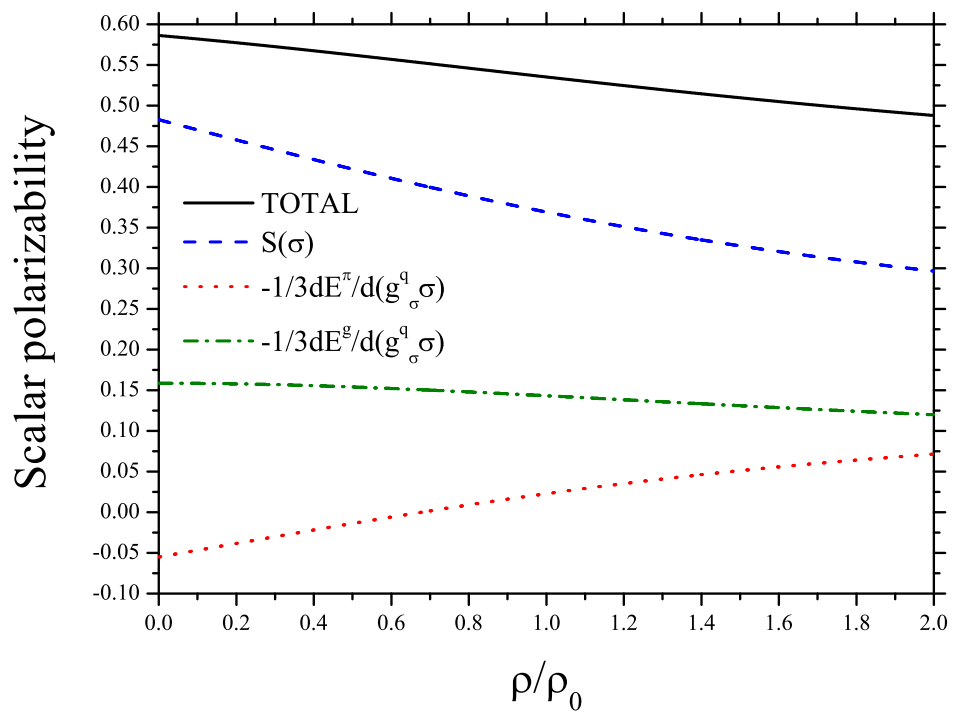

FIG. 2: Scalar polarizability in the CQMC model (for $R_{N}=0.8 \mathrm{fm}$ ). The dashed curve presents the scalar polarizability in the usual QMC model, while the solid one is for the CQMC model. The dotted (dot-dashed) curve shows the pion (gluon) contribution to the scalar polarizability.

In the CQMC model, the bag radius is swelled by a few percent at $\rho_{0}$. The quark eigenvalue, $x$, decreases by about $20 \%$, which leads to the smaller in-medium nucleon mass than in the QMC model. Although the rms radius of a nucleon swells by about $6 \%$ at $\rho_{0}$, it may still be within the experimental constraint [19].

In Fig. 2, we present the scalar polarizability in the CQMC model, which is given by a sum of the quark scalar density, $S(\sigma)$, and the contributions from the pion and gluon exchanges. We find that even in the CQMC model the scalar polarizability decreases with increasing $\rho_{B}$. Because of this reduction of the scalar polarizability in matter, the present 


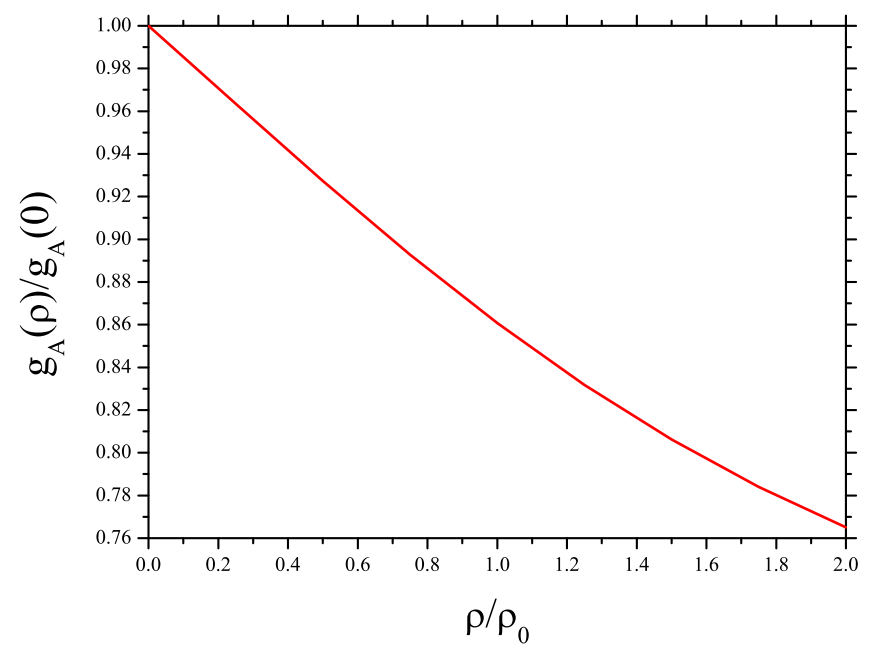

FIG. 3: Variation of $g_{A}$ in matter (for $R_{N}=0.8 \mathrm{fm}$ ).

model can achieve the nuclear saturation property with a much smaller value of the nuclear incompressibility, $K$, than in QHD.

In Fig. 3, as an example, we show the axial vector coupling constant, $g_{A}$, in matter. If the pion is massless, the pion pole term in the axial vector current gives just $1 / 3$ of the quark core contribution i.e., the MIT-bag-model value [10, 20]. However, when the pion is massive, the pion current does not contribute to $g_{A}$. Here the quark core contribution is thus calculated with the corrections due to the Peierls-Thouless projection method and the Lorentz contraction of the bag [20]. We obtain $g_{A}=1.15$ in free space. The CQMC predicts a reduction of about $14 \%$ for $g_{A}$ at $\rho_{0}$. Note that the pion field surrounding a nucleon becomes also weaker with increasing $\rho_{B}$ since the pion itself is mainly generated by the pv interaction.

In the present model, of importance is the scalar polarizability. It describes the response of a quark to the scalar field in matter and leads to the reduction of the $\sigma$-N coupling constant [1, 21]. Such response arises from the change of the quark wave function in a medium, and it is thus the many-body effect [8]. Then, the CQMC predicts that the iso-scalar, central force is weakened depending on $\rho_{B}$. On the other hand, in EFT, the (supposed) $\sigma$ exchange in the OBE model can be well understood by the correlated, two-pion contribution [22, 23]. However, recent calculations for matter show that, the $\mathrm{N}^{3} \mathrm{LO}$ potential based on EFT produces very deep overbinding at large $\rho_{B}$ [24]. Thus, this reduction of the 
central force may be favorable. To draw more definite conclusions, however, further studies are necessary.

In summary, we have developed for the first time a chiral version of the quark-meson coupling model based on the cloudy bag model, in which the effects of pion cloud and gluon exchange are included self-consistently. The model can describe a symmetric nuclear matter reasonably well. We have also shown that $g_{A}$ decreases with increasing $\rho_{B}$. This implies that the iso-scalar, central nuclear force should be weakened in matter. At $\mathcal{O}\left(1 / f_{\pi}^{2}\right)$, the CBM Lagrangian automatically provides the Weinberg-Tomozawa (WT) term, which is a new source of $g_{A}[20,25]$. Thus, in the future, it is desirable to perform a self-consistent

calculation up to $\mathcal{O}\left(1 / f_{\pi}^{2}\right)$ including the WT term. It is also very interesting to compare the CQMC with the EFT approach to investigate the internal structure change of the nucleon in medium.

\section{Acknowledgments}

The authors thank A.W. Thomas for valuable discussions on the pion-cloud effect. This work was supported by Academic Frontier Project (Holcs, Tokyo University of Science, 2005) of MEXT, and by the US Department of Energy, Office of Nuclear Physics, through contract no. DE-AC05-06OR23177, under which Jefferson Science Associates, LLC, operates Jefferson Lab.

[1] P.A.M. Guichon, Phys. Lett. B200 (1988) 235;

K. Saito, A.W. Thomas, Phys. Lett. B327 (1994) 9;

K. Saito, K. Tsushima, A.W. Thomas, Phys. Rev. C55 (1997) 2637; Prog. Part. Nucl. Phys. $58(2007) 1$

[2] For a recent review, R. Machleidt, nucl-th/0710.2940.

[3] For example, G.P. Lepage, nucl-th/9706029.

[4] R.D. Entem, R. Machleidt, Phys. Rev. C66 (2002) 014002; C68 (2003) 041001.

[5] M. Lutz, B. Friman, Ch. Appel, Phys. Lett. B474 (2000) 7;

S. Fritsch, N. Kaiser, W. Weise, Nucl. Phys. A750 (2005) 259. 
[6] S. Strauch (Hall A collaboration), Proc. of the Fifth Int. Workshop on Neutrino-Nucleus Interactions in the Few-GeV Region (NuInt07), nucl-ex/0709.4034.

[7] J.R. Smith, G.A. Miller, Phys,. Rev. Lett. 91 (2003) 212301; Phys. Rev. C 70 (2004) 065205.

[8] P.A.M. Guichon, H.H. Matevosyan, N. Sandulescu, A.W. Thomas, Nucl. Phys. A772 (2006) 1.

[9] A.W. Thomas, J. Phys. G7 (1981) L283; Adv. Nucl. Phys. 13 (1984) 1.

[10] S.A. Chin, Nucl. Phys. A382 (1982) 355.

[11] T. Inoue, V.E. Lyubovitskij, Th. Gutsche, A. Faessler, Int. J. Mod. Phys. E15 (2006) 121.

[12] A.W. Thomas, G. Krein, Phys. Lett. B456 (1999) 5;

D.B. Leinweber, A.W. Thomas, K. Tsushima, S.V. Wright, Phys. Rev. D61 (2000) 074502.

[13] T. DeGrand, R.L. Jaffe, K. Johnson, J. Kiskis, Phys. Rev. D12 (1975) 2060.

[14] K. Saito, Prog. Theor. Phys. (Kyoto) 71 (1984) 775.

[15] E.B. Dally et al, Phys. Rev. D24 (1981) 1718.

[16] S. Capstick, N. Isgur, Phys. Rev. D34 (1986) 2809.

[17] R.D. Young, D.B. Leinweber, A.W. Thomas, S.V. Wright, Phys. Rev. D66 (2002) 094507.

[18] J. Delorme, M. Ericson, P.A.M. Guichon, A.W. Thomas, Phys. Rev. C61 (2000) 025202.

[19] R.D. Mckeown, Phys. Rev. Lett. 56 (1986) 1452;

D.H. Lu, A.W. Thomas, K. Tsushima, A.G. Williams, K. Saito, Phys. Lett. B417 (1998) 217.

[20] K. Tsushima, T. Yamaguchi, Y. Kohyama, K. Kubodera, Nucl. Phys. A489 (1988) 557;

D.H. Lu, A.W. Thomas, K. Tsushima, nucl-th/0112001 (unpublished);

K. Tsushima, Hungchong Kim, K. Saito, Phys. Rev. C70 (2004) 038501.

[21] K. Saito, K. Tsushima, Phys. Lett. B575 (2003) 4.

[22] N. Kaiser, R. Brockmann, W. Weise, Nucl. Phys. A625 (1997) 758.

[23] J.F. Donoghue, Phys. Lett. B643 (2006) 165; Phys. Rev. C74 (2006) 024002.

[24] Z.H. Li, U. Lombardo, H.-J. Schulze, W. Zuo, L.W. Chen, H.R. Ma, Phys. Rev. C74 (2006) 047304;

P. Saviankou, S. Krewald, E. Epelbaum, Ulf-G. Meissner, nucl-th/0802.3782.

[25] M.A. Morgan, G.A. Miller, A.W. Thomas, Phys. Rev. D33 (1986) 817. 